\title{
A Hybrid Decision Making Model for Evaluating Land Combat Vehicle System
}

\author{
M.-T. Nguyen ${ }^{\mathrm{a}}$ \& $\quad$ T. Cao $^{\mathrm{a}}$ \\ a Defence Science and Technology Group, Australia \\ Email: \{Minh-Tuan.Nguyen/Thang.Cao\}@dst.defence.gov.au
}

\begin{abstract}
New Land Combat Vehicles (LCV) are being acquired to improve the fighting capability of the Australian Defence Force (ADF). Combat vehicle design has traditionally focussed on the use of new and improved technologies to provide an appropriate level of survivability and lethality against a range of threats. However, other factors such as sensors, firepower, mobility, tactics, situational awareness and communications also play a role in increasing or decreasing the survivability and lethality of LCV.
\end{abstract}

For the most part, LCV system interactions between the components are defined by logical or physical links which complicate the evaluation of its capabilities. In addition, the different types of operational scenarios and threats provide a challenge to the Decision Maker (DM) in providing a clear answer to the problem of determining the 'best' combination of LCV system configurations.

This paper utilises two methodologies, Bayesian Network (BN) and Multi-Criteria Decision Analysis (MCDA), and presents a hybrid decision making model for evaluating the operational impact of different LCV system specifications and configurations. $\mathrm{BN}$ is employed to establish a qualitative and quantitative representation of the relations between the variables of the model and calculate standard values of uncertain LCV capabilities like survivability, lethality, etc. MCDA is adopted to integrate the influence of LCV capabilities and calculate the utility value of the selected options.

In order to analyse a very large number of model outputs from all possible system components, a prototype tool of the hybrid approach has been developed. This tool conveniently performs inferences over the BN, examines various proposed configurations in a more intuitive and friendly fashion using data analysis, visualisation and sensitivity analysis techniques, and also searches for the 'best' configuration for the LCV system. The tool utilises publicrelease BN software (GeNIe), statistical packages (R) and a built-in Analytic Hierarchy Process (AHP) to allow the required calculations to be completed automatically and the results captured in both tabular and graphical form.

Keywords: Bayesian network, multi-criteria decision making, analytic hierarchy process, data visualisation, data analysis 


\section{INTRODUCTION}

The new close combat capability, known as the Land Combat Vehicle (LCV) system, is being acquired and will replace and enhance those mounted close combat capabilities currently enabled by the Protected Mobility Vehicle (PMV), Australian Light Armoured Vehicle (ASLAV) and Armoured Personnel Carrier (M113) fleets [Department of Defence, 2011]. Combat vehicle design has traditionally focussed on the use of new and improved technologies to provide an appropriate level of survivability and lethality against a range of threats. However, other factors such as sensors, firepower, mobility, tactics, situational awareness and communications also play a role in increasing or decreasing the survivability of the crew and the platform and, in turn, have a large impact on the operational effectiveness in the Land environment.

For the most part of LCV interactions between the components are defined by logical or physical links which complicate the evaluation of the dependability of these kinds of systems. In addition, the different types of operational scenarios and threats provide a challenge to Decision Maker (DM) in providing a clear answer to the problem of determining the 'best' combination of LCV system configurations.

Bayesian Network (BN) is a graphical probabilistic modelling technique suitable for knowledge-based evaluation systems. It enables us to model and reason about uncertainty, complexity and interdependencies between system components. BN has been widely used in many applications for dependability and risk analysis [Weber et al., 2012]; medicine, computing, natural sciences and engineering [Pourret et al., 2008]. For defence applications, BN has also been applied in reliability prediction for military vehicles [Neil et al., 2001]; military operational planning [Falzon, 2006] and a model of LCV system was constructed using BN to examine the operational impact of its different system configurations [Cao \& Chau, 2015] recently.

The Multi-Criteria Decision Analysis (MCDA) or Multi-Criteria Decision Making (MCDM) involves "making preference decisions" (such as evaluation, prioritisation, selection, and so on) over the available alternatives that are characterised by multiple, usually conflicting criteria (see [Hwang \& Yoon, 1981; Greco et al., 2016] for a full survey, also [Nguyen, 2003] for a cross-section of potentially useful methods in Defence).

This paper utilises BN and MCDA methodologies, and presents a hybrid decision making model for evaluating the operational impact of different LCV system specifications and configurations. BN is employed to establish a qualitative and quantitative representation of the relations between the variables of the model and calculate standard values of uncertain LCV capabilities like survivability, lethality, etc. MCDA is adopted to integrate the influence of LCV capabilities and DM's preferences, and calculate the overall utility value of the selected options.

In order to analyse a very large number of model outputs from all possible system components, a prototype tool of the hybrid model has been developed to conveniently perform inferences over the BN, to examine various proposed configurations in a more intuitive and friendly fashion using data analysis, visualisation and sensitivity analysis techniques, and also to search for the 'best' configuration for the LCV system. The tool is 'packaged' with public BN software SMILE (Structural Modelling, Inference, and Learning Engine) [Druzdzel, 1999], statistical packages R [R Core Team, 2015] and a built-in Analytic Hierarchy Process (AHP) [Saaty, 1980] to allow required calculations to be completely automated and results to be captured in both tabular and graphical form. The hybrid model is a continuation of the study on the LCV BN modelling [Cao \& Chau, 2015] and the recently development of its accompanied decision support tool [Nguyen et al., 2016].

\section{2 evaluating LCV by Operational Capabilities}

The purpose of an evaluating framework is to select LCV system specifications and configurations at the desired operational effectiveness. The measurement of each configuration option is determined by system capabilities [Department of Defence, 2011] that provides enhanced land combat survivability and lethality to LCV with superior protection 1, firepower 2 , mobility 3 , knowledge and connectivity 4 , so it is desirable to develop a MCDA model to support upcoming decisions for the ranking and selection of the "best" LCV. In MCDA, DMs choose their most preferred option(s) from a set of options that are evaluated in terms of weighting and utility values of the alternatives on the (sub-)criteria. The weights on these (sub-)criteria are based on the subjective DM's preferences.

\subsection{Criteria and Option Set}

Denote by $C \stackrel{\text { def }}{=}\left\{C_{i}, i=1, \ldots, n\right\}$ the set of $n$ LCV capabilities. We restrict attention to the following 4 types of LCV system capabilities for our illustration purpose: $\left(C_{1}\right)$ survivability; $\left(C_{2}\right)$ lethality; $\left(C_{3}\right)$ mobility and $\left(C_{4}\right)$ knowledge (or C4ISR5).

Each LCV capability is then divided into different types (called sub-capabilities) for our manageable assessment

\footnotetext{
${ }^{1}$ under enemy's offensive threats, e.g. Improvised Explosive Device (IED), Anti-Tank Guided Missile (ATGM), Rocket-Propelled Grenade (RPG)

${ }^{2}$ under enemy's defensive threats, e.g. Vehicle armour, Active Protection System (APS)

${ }^{3}$ highly effective close combat, tactical and operational mobility

${ }^{4}$ high situational awareness, battlespace knowledge, exercise command \& control and communication capability (C4ISR)

${ }^{5}$ refer to the concept of Command, Control, Communications, Computers, Intelligence, Surveillance and Reconnaissance.
} 
(for example, survivability under enemy's Improvised Explosive Device (IED), Anti-Tank Guided Missile (ATGM), Rocket-Propelled Grenade (RPG), etc). Let us denote by $C_{i} \stackrel{\text { def }}{=}\left\{C_{i j}, j=1, \ldots, n_{i}\right\}$ the set of LCV sub-capabilities for Capability $i$. These sub-capabilities $\left(C_{i j}\right)$ will be inferred from BN models described in Section 3 .

The selection process can be divided into two steps: the first step is to choose which system configuration should be altered/enhanced from the status quo/current option; the second is to decide which option in the available list should be evaluated. The option set for each step is deterministic. Let $p$ be the number of system configurations and $l_{k}$ the number of options in LCV configuration $k, k=1, \ldots, p$. In our illustrated example, we consider 15 system configurations (e.g. armour protection system, signature management system, cannon, missile, communication system, etc). Each system configuration has from 2 to 4 options (e.g. for communication system configuration, one can select one from 4 options: UHF, VHF_HF6, SATCOM7, or all of the above).

\subsection{Determining Weights of Criteria}

MCDA evaluates the influence of multiple criteria by calculating a utility value where each criterion takes a certain weight. A few methods have been introduced in determining the criterion weights including a scoring/rating method and pairwise comparison method (see e.g. [Nguyen, 2003] and reference therein). Both methods are used in this work and implemented in our decision support tool, Bayesian Network Analysis Tool (BNAT) (Section 5). Note that we have to determine the weight for both levels: Capability $C_{i}$ and Sub-capability $C_{i j}$ where $i=1, \ldots, n$ and $j=1, \ldots, n_{i}, \forall n \in \mathbb{N}, \forall n_{i} \in \mathbb{N}$.

Direct Rating/Scoring: the method is to rate each criterion according to the importance believed by DM and determine the weights according to the following equation $w_{c}=r_{c} / \sum_{i=1}^{s} r_{i}$, where $w_{c}$ is the weight of the criterion $c ; s$ is the number of decision criteria, and $r_{i}$ is the importance rating of criterion $i$ (in any scoring/rating scheme, for example $\left.r_{i} \in[1,10]\right)$. This method is often preferred by DMs because it is the easiest and simplest method to use. Unfortunately, the method can yield biased and inconsistent results.

Pairwise Comparison: the method is based on the Analytic Hierarchy Process (AHP) [Saaty, 1980]. All the elements of the same hierarchical level are compared as pairs with respect to the corresponding elements in the next higher level, obtaining a matrix of pairwise comparisons. For representing the relative importance of one element over another, a scale for pairwise comparisons is introduced [Saaty, 1988, 1992]. For this relative comparison the fundamental scale of Table 1 can be used. It allows the comparisons to be expressed in verbal terms which are then translated into the corresponding numbers.

In particular for the final LCV evaluation, a $n$-by- $n$ matrix $A$ of pairwise comparisons is constructed. The components $a_{i j}(i, j=1,2, \ldots, n)$ of the matrix $A$ are numerical entries, which express (by the pairwise comparison scale) the relative importance of the Capability $i$ over Capability $j$ with respect to the final LCV operational effectiveness. Thus the matrix $A$ has the form:

$$
\left(\begin{array}{cccc}
a_{11} & a_{12} & \ldots & a_{1 n} \\
a_{21} & a_{22} & \ldots & a_{2 n} \\
\ldots & \ldots & \ldots & \ldots \\
a_{n 1} & a_{n 2} & \ldots & a_{n n}
\end{array}\right) \quad \text { where } a_{i i}=1, a_{j i}=\frac{1}{a_{i j}}, a_{i j} \neq 0
$$

In order to calculate relative priorities (weights) among the $n$ elements of the matrix $A$, the principal eigenvector of the matrix is computed. Then this eigenvector is normalised obtaining the "priority vector". The AHP methods allows measurement of the consistency of an evaluation (AHP Consistency Ratio (CR) indicator, and as a rule of thumb, CR should not exceed $10 \%$ significantly) [Saaty, 2012]. If the inconsistency is too high, it is necessary to reformulate the judgements through new pairwise comparisons.

In BNAT, AHP is applied for group decision making situation as well. Therefore, it will be necessary to analyse individual judgements; and to find a measure of consensus for the aggregated group result. We use Shannon entropy [Jost, 2006] and its partitioning in two independent components (alpha and beta diversity) to derive an AHP consensus indicator. We then apply a geometric mean [Aull-Hyde et al., 2006] for aggregation between DM preferences rather than arithmetic mean. The AHP weighting method is preferred for use in BNAT because it allows measuring an evaluation consistency and consensus in group decision making.

\footnotetext{
${ }^{6}$ The International Telecommunication Union (ITU, www.Itu.int) designates for radio frequencies in the range Ultra high frequency (UHF) between $300 \mathrm{MHz}$ and $3 \mathrm{GHz}$; Very high frequency (VHF) from $30 \mathrm{MHz}$ to $300 \mathrm{MHz}$; High frequency (HF) between 3 and $30 \mathrm{MHz}$.

${ }^{7}$ The Wideband Global SATCOM system (WGS) is a high capacity satellite communications system planned for use in partnership by the United States Department of Defense and the Australian Department of Defence, http://www.defence.gov.au/AnnualReports/07-08/vol2/ch3_02_wgs.htm accessed on the 9 October 2017.
} 


\section{Assessing LCV Sub-capabilities Using BN Models}

For the most part of LCV interactions between the components are defined by logical or physical links which complicate the evaluation of the dependability of these kinds of systems. BN was identified to be very useful for assessing LCV sub-capabilities [Cao \& Chau, 2015] since they permit a qualitative and quantitative representation of the relations between the variables of the model.

\subsection{Bayesian Network}

$\mathrm{BN}$ is a graphical model for conceptualising and quantifying uncertainty about the causal relationships between variables and consists of

- a list of nodes representing random variables $\mathrm{X} \stackrel{\text { def }}{=}\left\{X_{1}, X_{2}, \ldots, X_{k}\right\}$,

- a list of directed links expressing causal probabilistic relationships between the variables, and

- Conditional Probability Tables (CPTs) $\left\{P\left(X_{i} \mid \Pi_{i}\right)\right\}$ detailing the strength of causal relationship between the nodes, where $\Pi_{i} \subseteq \mathrm{X} \backslash\left\{X_{i}\right\}$ is the parent node set of $X_{i}$.

The theory of the calculation probability in BN is inspired from Bayes' theorem and the joint probability distribution in $\mathrm{BN}$ can be calculated by the multiplication of the conditional probabilities of all the nodes

$$
p\left(X_{1}, X_{2}, \ldots, X_{k}\right)=\prod_{i=1}^{k} p\left(X_{i} \mid \Pi_{i}\right) .
$$

Establishment of the BN model contains three aspects of work: (1) build the network structure - set up the network nodes and links; (2) determine the network parameters - populate the CPTs; and (3) probability propagation - network inference, i.e. calculating the probability of each node given evidences.

\subsection{BN Model for LCV System}

We have used the BN package GeNIE 8 to construct the LCV system network. GeNIe provides a good graphical interface for designing and developing a network structure and also has various tools for its CPTs to be populated. It allows modularity in large and complex systems such as LCV system by using the special node type 'submodel'. The sub-model hosts sub-graphs of the entire graph and its structure can be examined in separation from the entire model. The LCV system model has 5 interacting sub-models:

- Survivability sub-model produces probability to survive various defensive threats

- Lethality sub-model produces probability to successfully engage various offensive threats

- Mobility sub-model produces probability to achieve highly effective close combat, tactical and operational mobility

- Knowledge/C4ISR sub-model produces probability to achieve high situational awareness, battlespace knowledge, exercise command \& control and communication capability

- Threat sub-model defines scenario, threats, linking defensive threats and offensive threat, acting as a linkage between other four sub-models

In particularly, the integrated survivability "onion' 9 (Figure 1) is applied for LCV survivability sub-model: in order to survive the threats, the following order of high level activities needed to be taken (each activity will depend on each threat and scenario): (1) Destroy enemy before engagement; (2) If enemy not destroyed, avoid detection; (3) If detected, avoid being targeted; (4) If targeted, avoid being hit; (5) If hit, avoid penetration, and (6) If penetrated, increase survive effect.

The BN model has been developed to identify the components of in-

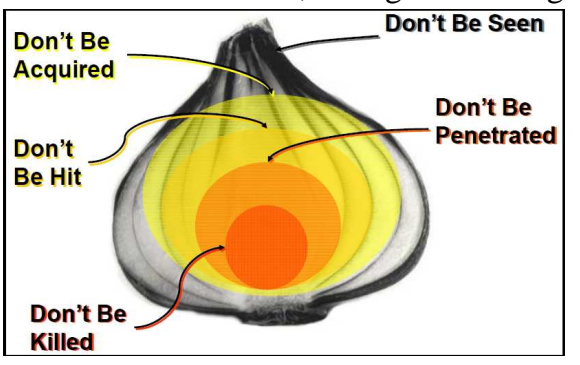

Figure 1. Integrated Survivability "Onion" terest and determine the interdependencies between them. Extensive interaction and elicitation workshops with DMs and Subject Matter Experts (SME) ensure a valid network structure and allow its CPTs to be populated. Figure 2 shows a BN modelling example of survivability under ATGM type $\mathrm{e}^{10}$. This diagram describes causal relationships among LCV configuration variables, whether it is on different terrain, etc., and whether LCV is survived under this type of missile whereas the strength of influence is quantified by the conditional probability table which is elicited from the experts. Here, the absence of a direct link between Configu-ration 11 and ATGM Survivability, for example, captures our understanding that there is no direct influence of this configuration on LCV survivability. The influence is mediated by the lethality effect of LCV weapon sub-system.

\section{Hybrid Model}

MCDA is a well known and popular technique in the evaluation of option selection problems because of its intuitiveness and ease of implementation. However, it has limitations that we take account of by using BN in a complementary way. Specifically, most MCDA techniques makes three critical assumptions that

\footnotetext{
${ }^{8}$ http://support.bayesfusion. com/docs/, accessed on the 9 October 2017.

9 http://handle.dtic.mil/100.2/ADA505271, accessed on the 9 October 2017.

${ }^{10}$ Note that the BN evaluation framework presented here do not represent an agreed or endorsed position by Defence.
} 


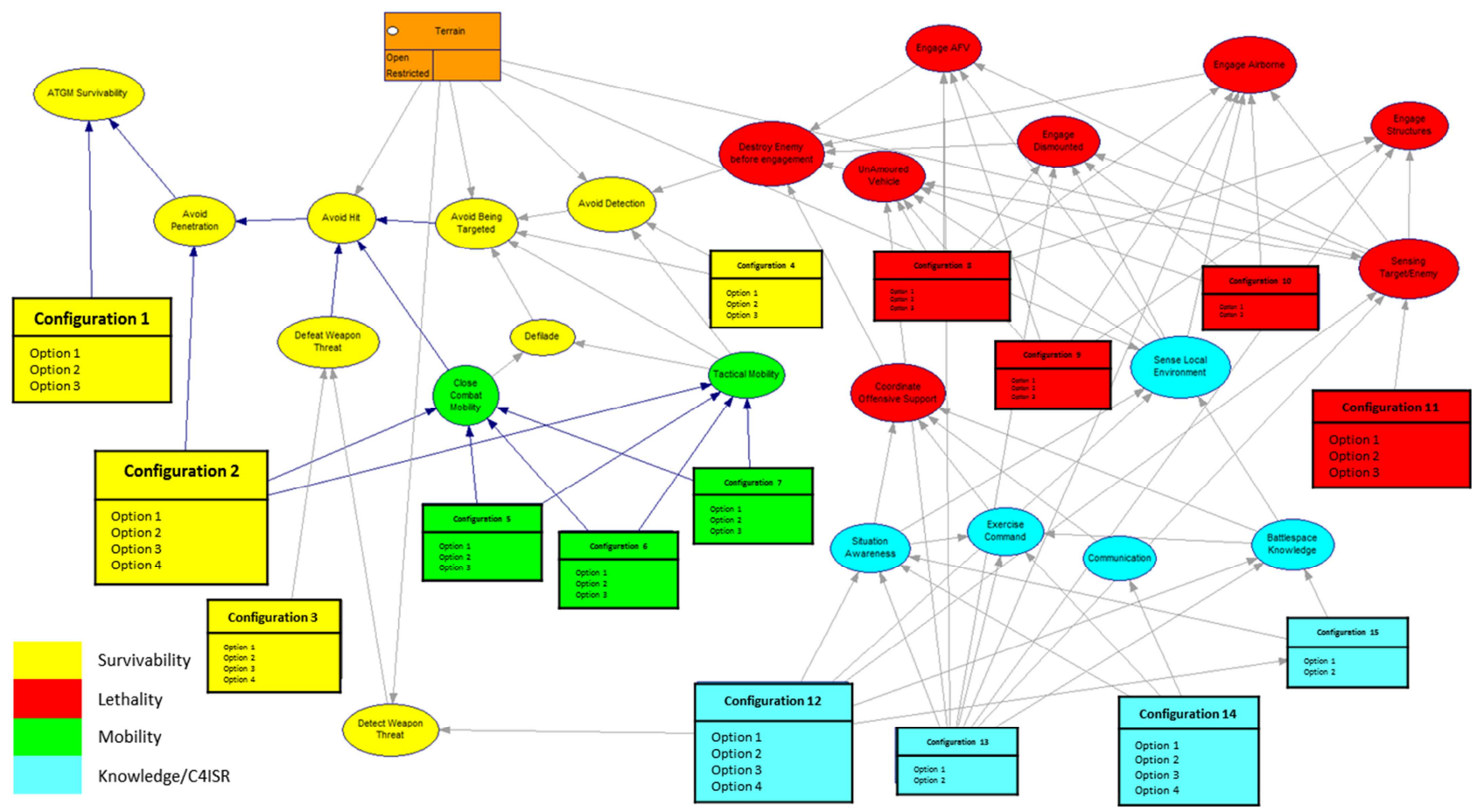

Figure 2. An Illustrated Example for Modelling LCV Survivability using BN

- the relevant criteria are well defined (e.g. for a given option $x$, it is obvious how to compute the value $v_{c}(x)$ for a given criterion $c$ );

- the relevant criteria are certain (e.g. the value $v_{c}(x)$ is deterministic rather than stochastic);

- the relevant criteria are independent of each other (i.e. the rank of an option does not depend on what other options there are and how many of them there may be).

Using MCDA alone is not applicable to our LCV system evaluation because the problem is large and complex, in particular containing a number of uncertainty situations caused by incomplete or noisy information, also having the nature of inter-dependency of LCV system configurations. Better decision making can be achieved if the uncertain interrelations among these decision elements can be explicitly modelled and reasoned with rather than ignored by some unrealistic assumptions or summarised out by subjective weighting schemas.

This paper utilises both BN and MCDA methodologies, and presents a hybrid decision making model for evaluating the operational impact of different LCV system specifications and configurations. BN is employed to establish a qualitative and quantitative representation of the relations between the variables of the model and calculate standard values of uncertain LCV capabilities like survivability, lethality, etc. MCDA is adopted to integrate the influence of LCV capabilities and calculate the utility value of the selected options.

Ranking scores are recursively defined by additive multi-attribute utility functions [Keeney \& Raiffa, 1976] and expected utility function [Neumann \& Morgenstern, 1953] where

$$
U(C) \stackrel{\text { def }}{=} \sum_{i=1}^{n} w_{i} U_{i}\left(C_{i}\right), \quad U_{i}\left(C_{i}\right) \stackrel{\text { def }}{=} \sum_{j=1}^{n_{i}} w_{i j} U_{i j}\left(C_{i j}\right), \quad \text { and } \quad U_{i j}\left(C_{i j}\right) \stackrel{\text { def }}{=} \sum_{k=1}^{s_{i j}} p_{k}^{i j} U_{k}^{i j}\left(C_{k}^{i j}\right)
$$

- $C_{i j}=\left\{C_{k}^{i j}, k=1, \ldots, s_{i j}\right\}$ is the set of states in the BN node of LCV Sub-capability $C_{i j}$ for any $i=1, \ldots, n$ and $j=1, \ldots, n_{i}$

- $\left\{w_{i} \mid \sum_{i=1}^{n} w_{i}=1\right\}$ and $\left\{w_{i j} \mid \sum_{j=1}^{n_{i}} w_{i j}=1\right\}$ are weighting constant sets derived from Analytic Hierarchical Process (AHP) priority vectors or from direct rating process (Section 2.2);

- $\left\{p_{k}^{i j} \mid \sum_{k=1}^{s_{i j}} p_{k}^{i j}=1\right\}$ is probability distribution for the BN node of LCV Sub-capability $C_{i j}$ (Section 3.1);

- $U_{k}^{i j}\left(C_{k}^{i j}\right)$ is the utility value of LCV Sub-capability $C_{i j}$ in State $k$.

\section{Decision Support Tool \& Illustrative Example}

Bayesian network software such as GeNIe provides a good graphical interface for model development and data capture. However, the intended end-user, who could be from a non-technical background, may not find such an interface appealing and comfortable to use. At times, comprehending such networks could be overwhelming, particularly when the number of nodes in the network is large. Furthermore, performing inferences on such a network could be difficult as it may not be straightforward to perceive how changes to a particular node value will affect the rest of the nodes it is connected to. Additionally, such a model requires sensitivity analysis and searching algorithms to confirm 
whether the best decision is sensitive to changes in the LCV configurations and utilities value and those capabilities are not readily available within a single tool.

\begin{tabular}{|c|c|c|c|c|}
\hline \multicolumn{5}{|c|}{ Unclassified } \\
\hline System Configuration & Your Option & $\begin{array}{l}\text { "Best" Option for } \\
\text { Open Terrain }\end{array}$ & $\begin{array}{l}\text { "Best" Option for } \\
\text { Restricted Terrain }\end{array}$ & Status Quo Option \\
\hline Config 1 & Opt 3 & opt 3 & opt 3 & Opt 1 \\
\hline Config 2 & Opt 3 & opt 3 & opt 3 & opt 1 \\
\hline Config 3 & Opt 2 & opt3 & opt 3 & Opt 1 \\
\hline Config 4 & Opt 1 & opt 3 & opt 3 & opt 1 \\
\hline Config 5 & Opt 1 & opt3 3 & opt 3 & Opt 1 \\
\hline Config 6 & Opt 3 & opt 3 & opt 3 & Opt 1 \\
\hline Config 7 & Opt 3 & Opt 3 & Opt 3 & opt 1 \\
\hline Config 8 & Opt 2 & Opt3 & Opt3 & Opt 1 \\
\hline Config 9 & Opt 2 & opt 2 & opt 2 & Opt 1 \\
\hline Config 10 & Opt 2 & opt 1 & opt 1 & Opt 1 \\
\hline Config 11 & Opt3 & Opt 3 & Opt 3 & Opt 1 \\
\hline Config 12 & Opt 3 & opt 4 & Opt 4 & Opt 1 \\
\hline Config 13 & Opt 4 & Opt 4 & opt 4 & opt 1 \\
\hline Config 14 & Opt 1 & opt 3 & opt 3 & Opt 1 \\
\hline Config 15 & Opt 1 & Opt 2 & Opt 2 & Opt 1 \\
\hline
\end{tabular}

(a) Option Selection

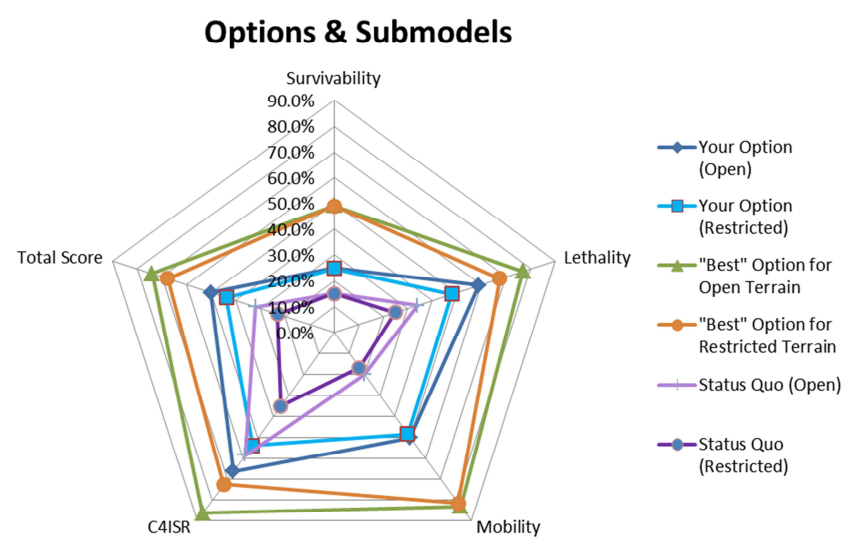

(b) Capability Option Comparison

Figure 3. Illustrated Example of User Interface in BNAT

To address the above-mentioned requirements and limitations, we develop the Bayesian Network Analysis Tool (BNAT) [Nguyen et al., 2016] which allows end-users to easily perform inferences over the $\mathrm{BN}$ as well as visualisation and comparison of results. The tool organises the nodes based on the causeand-effect relationship in the MCDM framework, i.e. BN nodes are classified into criteria or options. This makes the user-interaction more intuitive and user friendly. BNAT is a Microsoft Excel workbook 'packaged' with public software to allow required calculations to be completely automated and results to be captured in both tabular and graphical forms. Results from BNAT can be captured at any stage and export to other tools for different analysis types (e.g. Cost-Risk-Benefit Trade off analysis).

BNAT internally uses SMILE [Druzdzel,

\begin{tabular}{|c|c|c|c|c|}
\hline & & & & \\
\hline Evaluating Option & & & Your 0 & Option \\
\hline Output & & & Open & Restricted \\
\hline Survivability.Type1 & $1 \pm$ & 0.25 & $21.9 \%$ & $23.1 \%$ \\
\hline Survivability.Type2 & $\frac{5}{\frac{2}{0}}$ & 约 0.25 & $24.0 \%$ & $24.1 \%$ \\
\hline Survivability.Type3 & $\sum_{2}^{\infty}$ & 0.25 & $54.0 \%$ & $52.0 \%$ \\
\hline Survivability.Type4 & जั & 0.25 & $0.0 \%$ & $0.0 \%$ \\
\hline Engage.Type1 & & 0.20 & $77.1 \%$ & $65.4 \%$ \\
\hline Engage.Type2 & 로 & 0.20 & $22.5 \%$ & $15.5 \%$ \\
\hline Engage.Type3 & $\sum_{\bar{\pi}}^{\bar{\pi}}$ & 0.20 & $63.5 \%$ & $46.2 \%$ \\
\hline Engage.Type4 & 烏 & 0.20 & $53.3 \%$ & $44.2 \%$ \\
\hline Engage.Type5 & & 0.20 & $77.8 \%$ & $68.0 \%$ \\
\hline Mobility.TypeX & 로 & 0.33 & $60.0 \%$ & $60.0 \%$ \\
\hline Mobility.TypeY & $\overline{\overline{0}}$ & 0.33 & $50.0 \%$ & $50.0 \%$ \\
\hline Mobility.Typez & $\Sigma$ & 0.33 & $40.0 \%$ & $35.0 \%$ \\
\hline Knowledge Type 1 & & 0.20 & $25.0 \%$ & $25.0 \%$ \\
\hline Knowledge.Type2 & & 0.20 & $95.0 \%$ & $65.0 \%$ \\
\hline Knowledge.Type3 & $\frac{n}{y}$ & 0.20 & $67.1 \%$ & $49.9 \%$ \\
\hline Knowledge.Type4 & & 0.20 & $80.0 \%$ & $80.0 \%$ \\
\hline Knowledge.Type5 5 & & 0.20 & $63.7 \%$ & $48.6 \%$ \\
\hline
\end{tabular}

\begin{tabular}{|c|c|c|c|c|c|}
\hline \multicolumn{2}{|c|}{$\begin{array}{c}\text { "Best" Option for } \\
\text { Open Terrain }\end{array}$} & \multicolumn{2}{|c|}{\begin{tabular}{|l|} 
"Best" Option for \\
Restricted Terrain
\end{tabular}} & \multicolumn{2}{|c|}{ Status Quo Option } \\
\hline Open & Restricted & Open & Restricted & Open & Restricted \\
\hline $61.2 \%$ & $61.6 \%$ & $61.2 \%$ & $61.6 \%$ & $14.3 \%$ & $16.5 \%$ \\
\hline $61.7 \%$ & $61.8 \%$ & $61.7 \%$ & $1.8 \%$ & $13.5 \%$ & $14.4 \%$ \\
\hline $58.6 \%$ & $57.9 \%$ & $58.6 \%$ & $57.9 \%$ & $32.9 \%$ & $28.9 \%$ \\
\hline $14.4 \%$ & $14.2 \%$ & $14.4 \%$ & $14.2 \%$ & $0.0 \%$ & $0.0 \%$ \\
\hline $93.4 \%$ & $87.3 \%$ & 93.4\% & $87.3 \%$ & $40.8 \%$ & $27.7 \%$ \\
\hline $43.0 \%$ & $36.0 \%$ & $43.0 \%$ & $36.0 \%$ & $11.0 \%$ & $8.0 \%$ \\
\hline $88.5 \%$ & $70.5 \%$ & $88.5 \%$ & $70.5 \%$ & $40.0 \%$ & $30.0 \%$ \\
\hline $65.1 \%$ & $53.4 \%$ & $65.1 \%$ & $53.4 \%$ & $35.2 \%$ & $27.3 \%$ \\
\hline 94.1\% & $89.3 \%$ & $94.1 \%$ & $89.3 \%$ & $43.0 \%$ & $32.0 \%$ \\
\hline $90.0 \%$ & $90.0 \%$ & $90.0 \%$ & $90.0 \%$ & $10.0 \%$ & $10.0 \%$ \\
\hline $90.0 \%$ & $90.0 \%$ & $90.0 \%$ & $90.0 \%$ & $0.0 \%$ & $0.0 \%$ \\
\hline $70.0 \%$ & $65.0 \%$ & $70.0 \%$ & $65.0 \%$ & $50.0 \%$ & $40.0 \%$ \\
\hline $90.0 \%$ & $90.0 \%$ & $90.0 \%$ & $90.0 \%$ & $25.0 \%$ & $25.0 \%$ \\
\hline $95.0 \%$ & $65.0 \%$ & 95.0\% & $65.0 \%$ & $85.0 \%$ & $30.0 \%$ \\
\hline $88.8 \%$ & $66.8 \%$ & \begin{tabular}{|l|}
$88.8 \%$ \\
\end{tabular} & $66.8 \%$ & $63.5 \%$ & $30.5 \%$ \\
\hline $80.0 \%$ & $80.0 \%$ & $80.0 \%$ & $80.0 \%$ & $60.0 \%$ & $60.0 \%$ \\
\hline 78.4\% & $61.4 \%$ & 78.4\% & $61.4 \%$ & $58.3 \%$ & $30.2 \%$ \\
\hline
\end{tabular}

\begin{tabular}{|l|r|r|r|}
\hline \multirow{2}{*}{ Summary } & \multicolumn{3}{|c|}{ Setting Weights by AHP } \\
\hline Survivability & 0.25 & $25.0 \%$ & $24.8 \%$ \\
\cline { 2 - 4 } \\
\cline { 2 - 4 } \\
\cline { 2 - 4 } Mothality & 0.25 & $58.8 \%$ & $47.9 \%$ \\
\hline Mobility & 0.25 & $50.0 \%$ & $48.3 \%$ \\
\hline C4ISR & 0.25 & $66.2 \%$ & $53.7 \%$ \\
\hline
\end{tabular}

Figure 4. Illustrated Example of LCV System Ranking 1999] to perform the Bayesian inferences on the BN model developed. We have a built-in AHP to set weights for the criteria and data analysis is carried out by $\mathrm{R}$ statistical packages [R Core Team, 2015]. A sample of input options and output results is shown in Figure 3. BNAT makes users aware of the current/status quo option, also the best options using user's preferences from AHP or direct rating (Section 2.2). It also allows multiple user's options to be selected and its detailed sub-capability options to be compared (not shown in the figures). Ranking scores defined in Section 4 using Equation(2) are displayed in Figure 44.

The evaluation of any system configuration is produced within seconds on a PC i7-4770 CPU @ 3.4 GHz with 16 GB of RAM. However, searching for 'best' option will need all possible system configurations evaluated. BNAT deploys the computing power of the special $R$ packages $\left(f \mathrm{f}^{12}\right.$ and $f f b a s e^{13}$ ) for big data processing. With the illustrated example (more than 15 millions system configurations in total), it takes around 7 hours to calculate the marginal probability distributions for all $\mathrm{BN}$ nodes and to generate $4.54 \mathrm{~GB}$ data; Importing data into R is around 3 minutes, and searching options is about 30 second $\mathrm{s}^{14}$.

\section{Conclusion}

This paper develops and illustrates a hybrid decision making model for evaluating the operational impact of different LCV system specifications and configurations. It utilises both methodologies BN and MCDA. BN can explicitly model the uncertainty, complexity and inter-dependency of LCV system while MCDM transparently

\footnotetext{
${ }^{11}$ Data presented here is fictitious and used for illustrative purposes only.

${ }^{12}$ https://cran.r-project.org/web/packages/ff/index.html, accessed on the 9 October 2017.

${ }^{13}$ https://cran.r-project.org/web/packages/ffbase/index.html, accessed on the 9 October 2017.

${ }^{14}$ By separating the calculation of the marginal probability distributions from ranking option process, BNAT can quickly give system ranking scores when DM's preferences are changed.
} 
and conveniently examines various proposed LCV system configurations.

Note that in order to more fully appreciate the value of the proposed model and any case study, the analysis should be compared with the current evaluation approach adopted by Defence and the solutions it produces. Also significant verification and validation of the data model are required. What is clear, however, is that the model and data collection structures assist in making explicit the LCV configurations, scenario parameters and DM's preferences as well as providing a tool for automating/documenting some of the currently time-consuming evaluation processes.

\section{ACKNOWLedgments}

The authors would like to acknowledge Army Headquarters (AHQ) for supporting our study and providing Subject Matter Experts (SME) in various workshop. Our special thanks go to Land Mathematical Science (LMS) and Joint Warfare Mathematical Science (JWMS) groups for their advice on mathematical modelling aspects of our proposed model and analysis tool.

\section{REFERENCES}

Aull-Hyde, R., Erdogan, S., \& Duke, J. (2006). An experiment on the consistency of aggregated comparison matrices in ahp. European Journal of Operational Research, 171(1), 290-295.

Cao, T., \& Chau, W. (2015). Bayesian network model for analysing operational impact of land combat vehicle. In the 12th Engineering Mathematics and Applications Conference (EMAC). 6-9 December, Adelaide, Australia.

Department of Defence (2011). LAND 400: Land Combat Vehicle System - Concept of Operations. Version 3.0, UNRESTRICTED, Canberra, ACT, Australia.

Druzdzel, M. J. (1999). SMILE: Structural Modeling, Inference, and Learning Engine and GeNIe: A Development Environment for Graphical Decision-theoretic Models. In Proceedings of the 16th National Conference on Artificial Intelligence, AAAI '99/IAAI'99, (pp. 902-903). Menlo Park, CA, USA.

Falzon, L. (2006). Using bayesian network analysis to support centre of gravity analysis in military planning. European Journal of Operational Research, 170(2), 629 - 643.

Greco, S., Ehrgott, M., \& Figueira, J. R. (Eds.) (2016). Multiple Criteria Decision Analysis:State of the Art Surveys (International Series in Operations Research E Management Science). New York: Springer-Verlag, 2 ed.

Hwang, C.-L., \& Yoon, K. (1981). Multiple Attribute Decision Making: Methods and Applications A State-of-the-Art Survey. Berlin: Springer-Verlag Berlin Heidelberg.

Jost, L. (2006). Entropy and diversity. Oikos, 113(2), 363-375.

Keeney, R., \& Raiffa, H. (1976). Decisions with Multiple Objectives: Performances and Value Trade-Offs. New York: Wiley.

Neil, M., Fenton, N., Forey, S., \& Harris, R. (2001). Using bayesian belief networks to predict the reliability of military vehicles. Computing Control Engineering Journal, 12(1), 11-20.

Neumann, J. v., \& Morgenstern, O. (1953). Theory of Games and Economic Behavior. New York: Princeton University Press.

Nguyen, M.-T. (2003). Some Prioritisation Methods for Defence Planning. (DSTO-GD-0356), Defence Science and Technology Organisation, Australia.

Nguyen, M.-T., Cao, T., \& Chau, W. (2016). Bayesian network analysis tool for land combat vehicle system evaluation. In the 24th National Conference of the Australian Society for Operations Research (ASOR). 16-18 November, Canberra, Australia.

Pourret, O., Naim, P., \& Marcot, B. (2008). Bayesian Networks: A Practical Guide to Applications. Chichester, UK: Wiley.

R Core Team (2015). R: A Language and Environment for Statistical Computing. R Foundation for Statistical Computing, Vienna, Austria.

Saaty, T. (1988). Decision Making for Leaders. Pittsburgh: RWS Publications.

Saaty, T. (1992). Multicriteria Decision Making - The Analytic Hierarchy Process. Pittsburgh: RWS Publications.

Saaty, T. L. (1980). The Analytic Hierarchy Process. New York: McGraw-Hill.

Saaty, T. L. (2012). Decision Making for Leaders: The Analytic Hierarchy Process for Decisions in a Complex World. Pittsburgh: RWS Publications, third revised ed.

Weber, P., Medina-Oliva, G., Simon, C., \& Iung, B. (2012). Overview on bayesian networks applications for dependability, risk analysis and maintenance areas. Engineering Applications of Artificial Intelligence, 25(4), 671 682. Special Section: Dependable System Modelling and Analysis. 